\title{
Elucidating the Early Signal Transduction Pathways Leading to Fetal Brain Injury in Preterm Birth
}

\author{
MICHAL A. ELOVITZ, CONJEEVARAM MRINALINI, AND MARY D. SAMMEL \\ Department of Obstetrics and Gynecology [M.A.E., C.M.], Department of Biostatistics and Epidemiology [M.D.S.], University of \\ Pennsylvania, Philadelphia, Pennsylvania 19104
}

\begin{abstract}
Adverse neurologic outcome, including cerebral palsy, is a significant contributor to long-term morbidity in preterm neonates. However, the mechanisms leading to brain injury in the setting of a preterm birth are poorly understood. In the last decade, there has been a growing body of evidence correlating infection or inflammation with preterm birth. The presence of intrauterine inflammation significantly increases the risk for adverse neurologic outcome in the neonate. These studies were performed to elucidate the early signal transduction pathways activated in the fetal brain that may result in long-term neurologic injury. Using our mouse model of localized intrauterine inflammation, the activation of TH1/TH2 pathways in the placenta, fetus corpus, fetal liver, and fetal brain was investigated. Additional studies determined whether activation of TH1/TH2 pathways could promote cell death and alter glial development. Real-time PCR studies demonstrated that a robust TH1/TH2 response occurs rapidly in the fetal brain after exposure to intrauterine inflammation. The cytokine response in the fetus and placenta was not significantly correlated with the response in the fetal brain. Along with an immune response, cell death pathways were activated early in the fetal brain in response to intrauterine LPS. Implicating TH1/TH2 and cell death pathways in permanent brain injury are our findings of an increase in GFAP mRNA and protein as well as a loss of pro-oligodendrocytes. With increased understanding of the mechanisms by which inflammation promotes brain injury in the preterm neonate, identification of potential targets to limit adverse neonatal outcomes becomes possible. (Pediatr Res 59: 50-55, 2006)
\end{abstract}

$\mathrm{P}^{\mathrm{r}}$ reterm birth is a major cause of neonatal morbidity and mortality. In the United States, $10-12 \%$ of all pregnancies result in a preterm birth. Infection and/or inflammation is associated with many cases of what was previously described as "idiopathic" preterm birth (1). Inflammation, as defined by the presence of HCA, has been demonstrated to be present in the majority of early spontaneous preterm births (2). The existence of intrauterine inflammation in a preterm birth has significant clinical implications as preterm infants born in this setting have an increased risk for adverse outcomes $(3,4)$. More specifically, infants born from pregnancies complicated by HCA are at increased risk for neonatal brain abnormalities, such as PVL and intraventricular hemorrhage (IVH) (5-7).

Received March 8, 2005; accepted June 21, 2005.

Correspondence: Michal A. Elovitz, M.D., Center for Research on Reproduction and Women's Health, Department of Obstetrics and Gynecology, 421 Curie Blvd., 1353 BRB 2/3, Philadelphia, PA 19104-6142; e-mail: melovitz@obgyn.upenn.edu

This research was supported by a WRHR Award (K12) from University of Pennsylvania, the Bill and Melinda Gates Foundation, and R01 HD046544-01A1.

DOI: 10.1203/01.pdr.0000191141.21932.b6
Recent clinical data suggest that the presence of HCA in a preterm delivery increases the relative risk for subsequent development of $\mathrm{CP}(5,8)$. Even in the absence of PVL or white matter damage (WMD), some preterm infants will eventually be diagnosed with $\mathrm{CP}(9,10)$, suggesting that activation of inflammatory processes affect neurologic development even in the absence of overt structural damage.

Despite the growing body of evidence correlating intrauterine inflammation with adverse neurologic outcome including $\mathrm{CP}$, the precise mechanisms by which localized intrauterine inflammation promotes brain injury remains unclear. It is hypothesized that proinflammatory cytokines within maternal and fetal compartments contribute to neurologic damage in the preterm neonate (5). Biologically plausible mechanisms by which proinflammatory (TH1) cytokines can induce neuronal injury include the ability of TH1 cytokines to inhibit proliferation of neuronal precursor cells (11), evoke astrogliosis, a common finding in infants with PVL (12), and cause inflammation-induced oligodendrocyte loss via activation of cell death mediators (13). In addition, TH1 cytokines can alter the production of neurotrophins, a family of proteins that is involved in promoting neuronal and glial survival $(14,15)$. Whether activation of one or all of these pathways plays a mechanistic role in inflammation-induced brain injury in the preterm neonate is not clear.

We hypothesize that adverse neurologic outcome in the preterm neonate results from the activation of diverse signal transduction pathways culminating in both overt and subtle white matter damage. To understand, and ultimately decrease or prevent adverse neurologic outcome in the preterm neonate, we must elucidate the pathways and precise mechanisms by which localized intrauterine inflammation promotes brain injury. To this end, we have developed a mouse model of localized intrauterine inflammation that consistently results in preterm delivery with no maternal mortality $(16,17)$. Using this model, the studies described herein investigate the potential mechanisms by which intrauterine inflammation provokes brain injury. Our aims were to determine whether 1) intrauter-
Abbreviations: apaf-1, apoptotic protease-activating factor; BDNF, brainderived neurotrophic factor; CNTF, ciliary neurotrophic factor; CP, cerebral palsy; GFAP, glial fibrillary acidic protein; GNDF, glial cell-derived neurotrophic factor; HCA, histological chorioamnionitis; LPS, lipopolysaccharide; MGB, minor grove binder; PDGF, platelet-derived growth factor; PLP, proteolipid protein; PVL, periventricular leukomalacia 
ine inflammation activates a $\mathrm{TH} 1 / \mathrm{TH} 2$ response in the fetal brain, 2) the cytokine profile in the fetus and/or placenta could predict the inflammatory response in the fetal brain, 3) activation of an immune response in the fetal brain is associated with activation of cell death pathways, 4) intrauterine inflammation promotes changes in the production of neurotrophic factors, and 5) activation of these diverse signal transduction pathways resulted in disruption of normal glial development in the preterm fetal brain.

\section{MATERIALS AND METHODS}

Animals. CD-1 out-bred, timed-pregnant mice were purchased from Charles River Laboratories (Wilmington, MA). Animals were acclimated in our facility for 3-7 d before use in these experiments. All of the experiments performed were performed in accordance with the National Institutes of Health Guidelines on Laboratory Animals and with approval from the University of Pennsylvania's committee on Animal Use and Care.

Mouse model of localized intrauterine inflammation. Surgery was performed on day 15 of gestation as previously reported $(16,17)$ Day 15 is $79 \%$ of the CD-1 gestation, which corresponds to about 28-29 wk in the human, a time period of clinical interest. Briefly, using isoflurane anesthesia, the right uterine horn was exposed through a mini-laparotomy. LPS $(250 \mu \mathrm{g} / \mathrm{mouse}$ in $100 \mu \mathrm{L}$ ) from Escherichia coli (L2880, Sigma Chemical Co., St. Louis, MO) or sterile saline $(100 \mu \mathrm{L})$ was then infused into uterine horn with care not to enter the amniotic cavity, as previously described $(16,17)$. Previous experiments have demonstrated that animals receiving anesthesia, surgery, and intrauterine infusion with saline do not have preterm delivery and have similar number of live pups as control animals who receive no interventions $(16,17)$. For these studies, control animals received anesthesia, surgery, and intrauterine saline. Because our aim was to investigate the early signal transduction pathways involved in fetal brain injury, all specimens were harvested $6 \mathrm{~h}$ after intrauterine saline or LPS. From our prior work, we know that, at this time point, an inflammatory reaction is present in the uterus and cervix but preterm labor had not occurred $(16,17)$.

On day 15 of gestation, $6 \mathrm{~h}$ after intrauterine infusion of saline or LPS, fetal tissue and placentas were collected from the top two gestational sacs in the left uterine horn (the area most remote from LPS injection). The dam was kept under anesthesia while the pups were harvested to limit any confounding effect of uterine hypoperfusion (as would occur with $\mathrm{CO}_{2}$ asphyxiation). After adequate anesthesia was obtained, in both treatment groups, pups were harvested within 2 min of laparotomy. Pups in the left upper horn were alive at the time of harvest. Six hours after exposure to intrauterine saline or LPS, the following specimens were collected: 1) fetal corpus (whole fetal body without fetal head), 2) fetal liver, 3) fetal brain, and 4) placentas (fetal membranes were dissected off before processing). All tissues were processed for molecular studies as described below. To control for inherent genetic variability in our out-bred strain of mice, each fetal and placental sample is from a different dam.

Quantitative RT-PCR. Quantitative PCR (QPCR) was performed on fetal tissues and placentas to investigate gene expression of TH1/TH2 mediators. Additional QPCR experiments were performed on fetal brains using primers to caspase-1, -3, -8, and -9, apaf-1, GFAP, PLP, and nestin. RNA was harvested from tissues using Trizol (Invitrogen, Carlsbad, CA) and cDNA was generated using random hexamers. Specific primers, conjugated to an MGB probe, were purchased from Applied Biosystems (Foster City, CA). QPCR reactions were carried out using equivalent dilutions of each cDNA sample on the Applied Model 7900 sequence detector PCR machine (PE Applied Biosystems) as previously reported $(16,17)$. The relative abundance of the target was divided by the relative abundance of $18 \mathrm{~S}$ in each sample to generate a normalized abundance for the target transcript. All samples were analyzed in triplicate.

Quantification of neurotrophins. To determine whether intrauterine inflammation had an adverse effect on neurotrophins, we investigated mRNA expression of GNDF, PDGF, BDNF, and CNTF using specific primers and methods as described above for QPCR

Western blot analysis. To determine the protein expression of caspase-3, apaf-1, PLP/DM-20, and GFAP, Western blot analysis was performed using tissue homogenates from fetal brains harvested $6 \mathrm{~h}$ after intrauterine infusion of saline or LPS. Tissues were homogenized and the amount of crude protein present in each sample was determined using the BCA protein assay (Pierce Biotechnology, Inc., Rockford, IL). Forty micrograms of protein were mixed with $2 \times$ SDS sample buffer and subjected to SDS-PAGE. The separated proteins were transferred electrophoretically to PVDF membranes. Membranes were blocked in tris-buffered saline with 5\% nonfat dried milk powder for $2 \mathrm{~h}$. For caspase-3, an antibody that recognizes caspase-3 protein ( $35 \mathrm{kD})$ and activated caspase-3 (19 kD) was used in a 1:100 dilution (\#9662, Cell Signaling Technology, Beverly, MA). An anti-rabbit secondary antibody was used in a 1:15,000 dilution. For apaf-1, the antibody (Cell Signaling Technology) used recognizes the $100 \mathrm{kD}$ protein and splice variants in the $120-140 \mathrm{kD}$ range (primary antibody dilution 1:1000, anti-rabbit secondary in 1:15,000). For GFAP (Chemicon International, Temecula, CA), a 1:1000 dilution was used with an anti-mouse secondary antibody at 1:10,000. For PLP, the antibody (in 1:50, generously provided by Dr. Judith Grinspan) recognizes both the 26 and $20 \mathrm{kD}$ PLP and DM-20 protein, respectively. Blots were incubated overnight with the primary antibody, incubated for $1 \mathrm{~h}$ with the secondary antibody and then developed with the ECL system (Amersham Pharmacia Biotech, Piscataway, NJ) to detect antibody-antigen complexes. Protein expression was quantified using the National Institutes of Health imaging software using three to six samples from each treatment group.

Statistical methods. For each QPCR and Western blot experiment, three to nine samples (from different dams) for each treatment group were used. Statistical analysis was performed using the SigmaStat software (SPSS Inc., Chicago, IL) by one-way ANOVA and pair-wise comparison by StudentNewman-Keuls method (SNK) or by $t$ test for normally distributed data. For data that were not normally distributed, ANOVA on ranks with pair-wise comparison by Dunn's method or Mann-Whitney rank sum test were used. Pearson correlation coefficients were computed to evaluate the degree of association in the cytokine response between the different tissue compartments (placenta, fetus corpus, fetal liver, and fetal brain). The cytokine response was differentiated into a proinflammatory response (IL- $1 \beta$, TNF- $\alpha$, IFN- $\gamma$ ) and an anti-inflammatory response (IL-10, IL-4, IL-13, and IL-6).

\section{RESULTS}

Localized intrauterine inflammation activates a TH1 and TH2 response in the fetal brain. Using QPCR, we investigated 16 genes involved in TH1 or TH2 that have either been i) demonstrated to directly evoke brain injury in animal models or ii) implicated in adverse outcomes with preterm birth (Table 1). Fifteen of 16 genes investigated were significantly up-regulated in fetal brains exposed to intrauterine LPS, with 12/16 mediators being increased 2-fold or more in LPSexposed fetal brains.

Table 1. Activation of TH1/TH2 pathways in the fetal brain and corpus in response to intrauterine inflammation $(6 h)$

\begin{tabular}{lcrcc}
\hline TH1/TH2 & Brain* & $p$ value $\dagger$ & Fetal corpus* & $p$ value $\uparrow$ \\
\hline IL-2 & 2 & $0.030 \dagger$ & 6.5 & $0.002 \S$ \\
TCCR & 1.4 & $0.003 \dagger$ & 2 & $0.011 \dagger$ \\
CD28 & 2.9 & $<0.001 \dagger$ & 1.7 & $0.022 \dagger$ \\
IRF-1 & 1.6 & $0.004 \dagger$ & 1.7 & $0.002 \dagger$ \\
T-bet & 5 & $0.010 \dagger$ & 2.1 & $0.020 \dagger$ \\
Stat1 & 2.2 & $0.002 \dagger$ & 1.7 & $0.174 \dagger$ \\
Stat4 & 1.6 & $0.083 \S$ & 0.7 & $0.174 \S$ \\
TNF & 3.9 & $0.005 \dagger$ & 2.9 & $0.003 \dagger$ \\
IL-1 $\beta$ & 3.2 & $0.011 \dagger$ & 2.1 & $0.044 \S$ \\
GM-CSF & 2.4 & $0.010 \dagger$ & 3.4 & $0.046 \dagger$ \\
IFN- $\gamma$ & 1.6 & $0.033 \dagger$ & 1 & 0.911 \\
IL-15 & 2.3 & $0.001 \S$ & 4.6 & $<0.001 \dagger$ \\
IL-6 & 2.2 & $0.044 \S$ & 1.7 & $0.215 \dagger$ \\
IL-10 & 3.7 & $<0.001 \dagger$ & 6.5 & $0.008 \S$ \\
IL-4 & 18 & $0.007 \S$ & 13 & $0.006 \S$ \\
IL-13 & 5.5 & $0.035 \dagger$ & 1.5 & $0.281 \S$ \\
\hline
\end{tabular}

* Fold increase in gene expression in LPS-treated/saline treated; $n=3-6$ samples per treatment group. TCCR, t-cell receptor; IRF, interferon regulatory factor; IFN- $\gamma$, interferon- $\gamma$; GM-CSF, granulocyte-macrophage colonystimulating factor.

Statistical analysis comparing mean mRNA expression between two treatment groups: $\dagger t$ test; $\S$ analysis by Mann-Whitney rank sum. 
Inflammatory response in the fetal brain does not mimic what is occurring in the fetus. Observational studies have suggested that a fetal inflammatory response is the mechanism by which fetal brain injury occurs in inflammation-induced preterm birth (18). Therefore, to determine whether the potent TH1/TH2 response observed in the fetal brains exposed to LPS was directly related to a cytokine response in the fetus proper. We investigated the same 16 genes in the fetus corpus (Table 1). Whereas mRNA for $11 / 16$ genes were significantly up-regulated in the fetus corpus, only 9/16 were increased 2-fold or more.

We also assessed whether the cytokine response in either the fetal liver or placenta mirrored the response in the fetal brain (Table 2). For the placental and liver studies, we investigated those mediators that were the most dramatically elevated in the fetal brain. Intrauterine LPS resulted in a dramatic elevation of both $\mathrm{TH} 1$ and $\mathrm{TH} 2$ mediators from the placenta, with IL-10, a key TH2 cytokine being almost 100-fold increased in placentas from LPS-treated dams (Table 2). Fold increases in cytokine levels were the most elevated in placental tissue (Table 2). Between the different tissues, there was significant variability in the LPS-induced up-regulation of cytokine levels. Statistical analysis failed to demonstrate a significant correlation between the cytokine response in the placenta and fetal tissues, the fetus corpus and fetal brain, or fetal liver and fetal brain (data not shown). Of note, 10 mediators were more elevated in the brain than in the fetus corpus.

Cell death pathways are activated in fetal brains in response to intrauterine inflammation. Because IL-1 $\beta$ and TNF- $\alpha$ have been demonstrated in vitro to induce neuronal cell death via activation of several of the caspases $(19,20)$, we explored the involvement of caspase genes in inflammationinduced fetal brain injury. Four members of the caspase gene family were investigated, all of which were significantly increased in the fetal brains exposed to intrauterine LPS compared with saline (Fig. 1). In addition, caspase-3 protein was elevated in fetal brains exposed to LPS (Fig. 2). Because caspase- 3 activation appears to be dependent on apaf-1, we also investigated the effect of intrauterine inflammation on

Table 2. Fold increase in mRNA expression of TH1/TH2 genes between saline and LPS-treated animals

\begin{tabular}{lcccc}
\hline TH1/TH2 & Placenta & Fetal corpus & Fetal liver & Fetal brain \\
\hline IL-1 $\beta$ & 19.2 & 2.1 & 7.9 & 3.2 \\
TNF- $\alpha$ & 10.9 & 2.9 & 2.4 & 3.9 \\
IL-10 & 99.5 & 6.5 & 2.7 & 5.5 \\
IFN- $\gamma$ & 2.5 & 1 & 3.4 & 1.6 \\
IL-13 & 12.1 & 1.5 & 1.6 & 5.5 \\
IL-4 & 8 & 13 & 2.6 & 18 \\
IL-6 & 13 & 1.7 & 15.7 & 2.1 \\
CD-28 & 8.9 & 1.7 & 17.7 & 2.9 \\
\hline
\end{tabular}

Placentas and fetuses were harvested from left upper uterine horn. Fetus corpus is the whole fetus without the fetal head. The fetus corpus and fetal liver specimens were collected from different sets of experiments. The fetal liver was dissected from the fetal body. The fetal brain was dissected from the cranium. Three to six samples per treatment group were used for analysis. All values for the placenta, fetal liver, and fetal brain were statistically significant $(p<0.05) . p$ Values for the fetus are listed in Table 1.

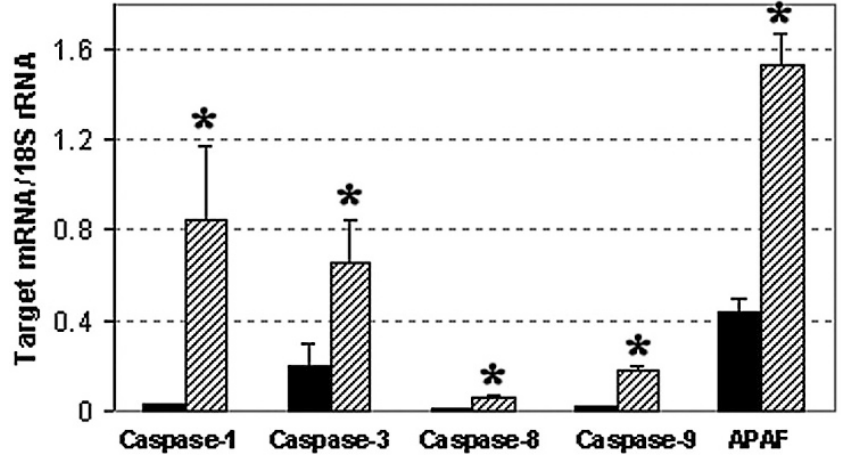

Figure 1. Quantitative PCR for mediators of cell death. Bars represent means \pm SE for each target ( $n=6$ for saline and $n=9$ for LPS). Statistical analysis demonstrated that caspase- $1,-3,-8$, and $-9\left({ }^{*} p \leq 0.001\right.$, Mann-Whitney rank sum for all caspases) mRNA expression was significantly elevated in LPSexposed fetal brains. APAF mRNA was also significantly increased ( $p \leq$ $0.001, t$ test) in LPS-exposed fetal brains (shaded bars) compared with saline (solid bars).
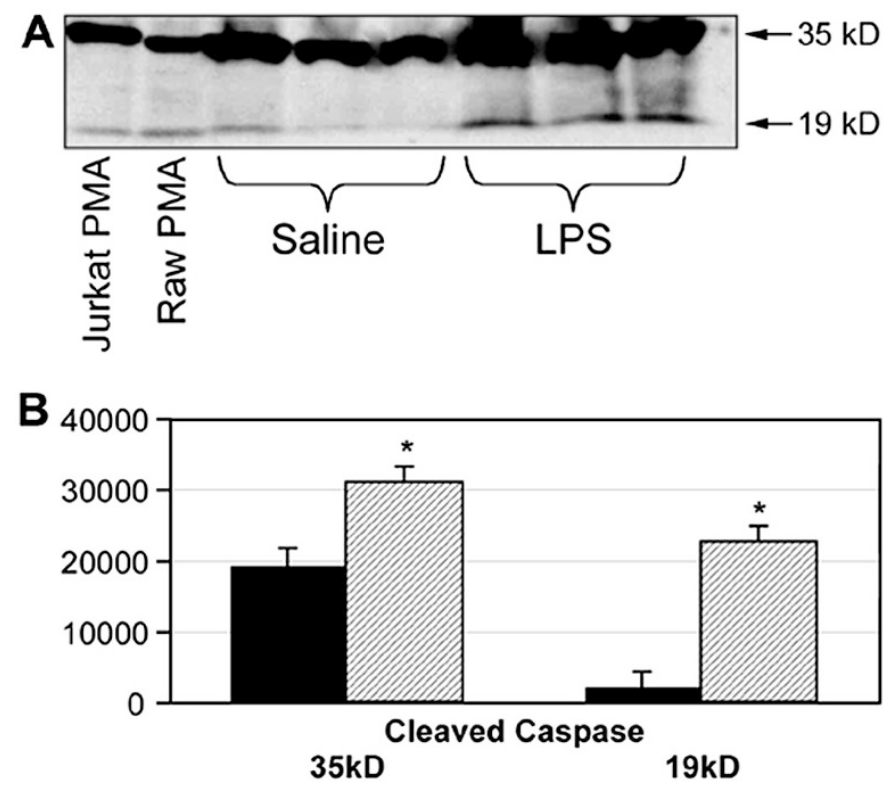

Figure 2. Caspase-3 protein expression. (A) Representative Western blot. Lanes 1 and 2: 1-Jurkat and RAW cell lysates stimulated with PMA; lanes 3-5: saline-exposed fetal brains; lanes 6-8: LPS-exposed fetal brains. Bands are visualized at $35 \mathrm{kD}$ (caspase-3 protein) and $19 \mathrm{kD}$ (cleaved caspase-3 protein). (B) Band density was quantified using National Institutes of Health imaging software. Means and SE were generated and depicted on the graph. An increase in the $35 \mathrm{kD}$ (caspase-3 protein) and $19 \mathrm{kD}$ (cleaved caspase-3 protein) was observed in fetal brains exposed to intrauterine inflammation (shaded bars) compared with shams (solid bars). $p=0.02$ and $p=0.002$, respectively, $t$ test.

apaf-1 expression in fetal brains. Intrauterine LPS resulted in a significant increase in both apaf-1 mRNA and protein expression in fetal brains exposed to intrauterine LPS (Figs. 1 and 3).

Exposure to intrauterine inflammation increases several neurotrophic factors. Because there is a paucity of data regarding the effect of intrauterine inflammation on other factors in the CNS that could modulate neuronal development, we investigated several neurotrophic factors known to be involved in glial development. We found that mRNA expression of select neurotrophins, essential for glial development 
A

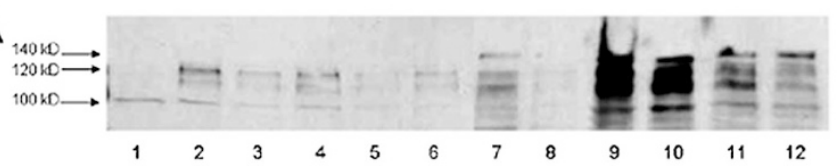

B

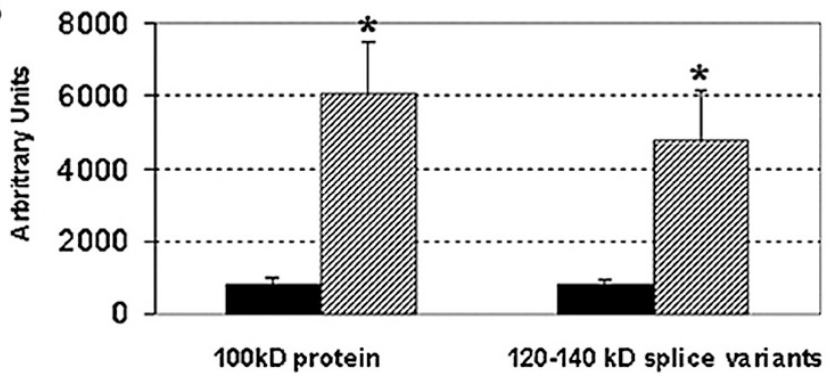

Figure 3. Apaf-1 protein expression. (A) Representative Western blot. Lane 1: RAW cell lysates stimulated with PMA; lanes 2-6: saline-exposed fetal brains; lanes 7-12: LPS-exposed fetal brains. Apaf-1 protein is at $100 \mathrm{kD}$, which is visualized in lane 1 as well as splice variants of apaf- 1 in the $120-140 \mathrm{kD}$ range. $(B)$ Band density was quantified using National Institutes of Health imaging software. Means and SE are depicted on the graph $(n=6)$. An increase in the $100 \mathrm{kD}$ and $120-140 \mathrm{kD}$ apaf-1 protein was observed in fetal brains exposed to intrauterine LPS (shaded bars) compared with saline (solid bars). $p=0.004$ and $0.015, t$ test, respectively.

and maturation, were all significantly increased in fetal brains exposed to intrauterine LPS (Fig. 4).

Activation of inflammatory pathways results in a reactive astrocytosis and a decline in pro-oligodendrocytes. Abnormal proliferation of astrocytes and a loss of oligodendrocytes are believed to contribute to white matter damage in the preterm infant $(21,22)$. Astrogliosis can be assessed by the amount of GFAP that is present in the brain (12). Because mature oligodendrocytes are not present in the mouse brain until the postnatal period (23), a marker of immature or prooligodendrocytes is necessary to determine whether intrauterine LPS can affect the development of this cell line. PLP is the major myelin protein of the CNS. DM-20 is produced by alternative splicing of the PLP gene and is expressed earlier in gestation than PLP (24). DM-20 and PLP are expressed in E12 mice brains and DM-20 serves as a marker of mature and immature oligodendrocytes (25). Nestin, an intermediate filament protein expressed early in neurodevelopment, is increased in the presence of brain injury and correlates with

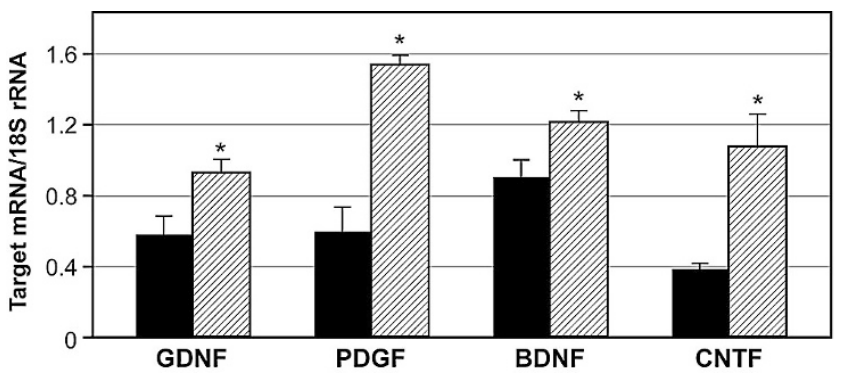

Figure 4. Message expression of neurotrophins. Bar graph depicting results of QPCR. Each bar represents means and SE for three to six fetal brains. GDNF, PDGF, BDNF, and CNTF mRNA were all significantly increased (*) in fetal brains exposed to intrauterine LPS (shaded bars) compared with sham (solid bars). $p=0.02$ ( $t$ test), $p=0.02$ (Mann-Whitney rank sum), $p=0.013$ ( $t$ test), and $p=0.006$ ( $t$ test), respectively. reversion to a more immature neural phenotype (26). Using QPCR, we demonstrated that GFAP and nestin mRNA were both significantly increased in fetal brains exposed to intrauterine inflammation (Fig. 5). In contrast, PLP mRNA was significantly decreased in fetal brains exposed to intrauterine LPS (Fig. 5). Glial development was significantly altered at the protein level as GFAP protein expression was significantly increased in LPS-exposed fetal brains (Fig. 6). Using an established antibody to PLP/DM-20 (27), we also demonstrated that expression of both the 20 and $26 \mathrm{kD}$ protein is significantly decreased in LPS-exposed fetal brains (Fig. 7). The same fetal brain specimens were used for the PLP and GFAP Western blot analysis (Figs. 6 and 7).

\section{DISCUSSION}

By using a model of localized intrauterine inflammation, we are able to closely approximate what occurs clinically in many cases of preterm birth. With this model, preterm delivery occurs by $20 \mathrm{~h}(16,17)$. We harvested fetal brains from dams $6 \mathrm{~h}$ after intrauterine infusion of LPS to determine the early signal transduction pathways triggered in the developing fetal brain. At this time point, proinflammatory cytokines are increased in both the uterus and cervix (16). We hypothesized that the increased production of cytokines in maternal compartment would be sufficient to activate inflammatory pathways in the fetal brain. These studies demonstrated, that early after intrauterine infusion of LPS, that a potent TH1/TH2 response is triggered in the fetal brain. Associated with this robust immune response was the activation of cell death pathways, proliferation of astrocytes and loss of oligodendrocyte precursors.

Various animal models have been created in an effort to elucidate the mechanisms by which intrauterine inflammation results in adverse neurologic outcome. Similar to others, our model is one of localized intrauterine inflammation which attempts to more accurately mimic what is observed in human preterm birth $(17,28-30)$. Some models in this group do not result in preterm delivery $(29,30)$. Signal transduction pathways involved in the emptying of the uterus and/or labor itself may have an affect on the fetus and the fetal brain. Our model elicits an inflammatory response in the uterus as well as causing preterm delivery $(16,17)$ and, therefore, serves as a

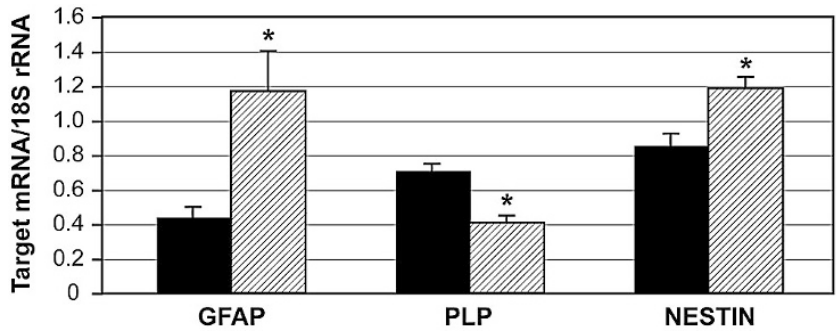

Figure 5. Effect of intrauterine inflammation on fetal brain. Bar graph depicting results of QPCR for GFAP, PLP, and nestin. Each bar represents means and SE for three to six fetal brains. GFAP and nestin were significantly increased (*) in fetal brains exposed to intrauterine LPS (shaded bars) compared with sham (solid bars) ( $p=0.03$ and $p=0.02$, respectively, $t$ test), whereas PLP was significantly decreased ( $p \leq 0.001, t$ test). 

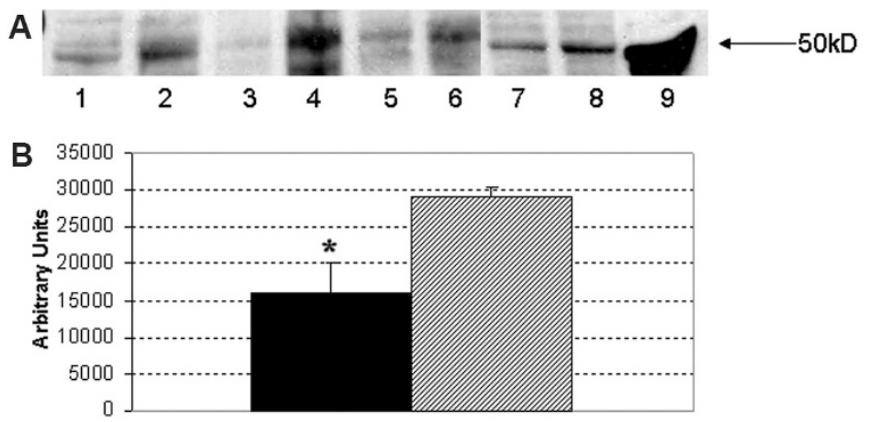

Figure 6. GFAP protein expression. (A) Western blot of GFAP protein expression in fetal brains. Lanes 1, 3, 5, and 7: fetal brains from saline-treated dams; lanes 2, 4, 6, 8: fetal brains from LPS-treated dams; lane 9 is adult mice brain extract (positive control) with the expected $50 \mathrm{kD}$ band for the GFAP protein. $(B)$ Band density was quantified using National Institutes of Health imaging software. Means and SE are depicted on the graph $(n=4$ per treatment group). An increase in the $50 \mathrm{kD}$ protein was observed in fetal brains exposed to intrauterine LPS (shaded bars) compared with saline (solid bars). $* p=0.02, t$ test.

A

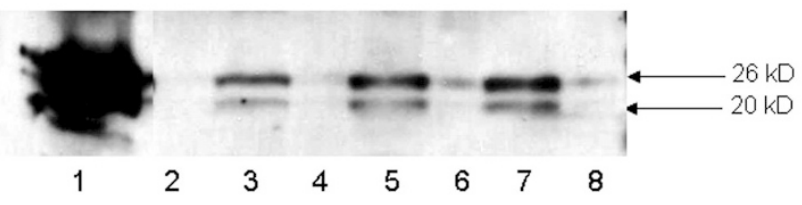

B

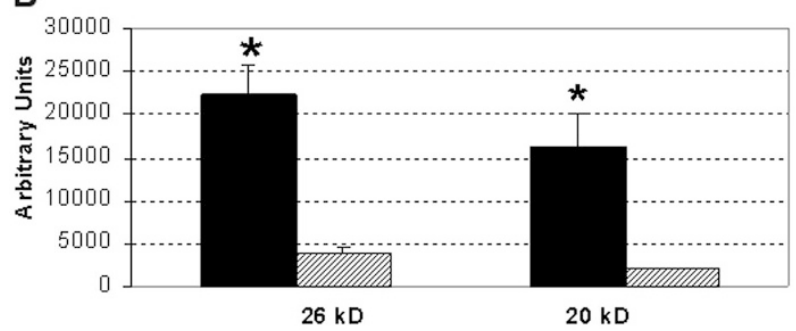

Figure 7. PLP/DM-20 protein expression. (A) Western blot of PLP/DM-20 protein expression in fetal brains. Lane 1 is adult mouse brain; lanes 3,5 , and 7: saline-exposed fetal brains; lanes 2, 4, 6, and 8: LPS-exposed fetal brains. (Same fetal brain specimens were used for GFAP blot, Fig. 6). (B) Band density was quantified using National Institutes of Health imaging software. Using three to four samples per treatment group, means and SE are depicted on the graph. An increase in the 20 and $26 \mathrm{kD}$ protein was observed in fetal brains exposed to intrauterine LPS (shaded bars) compared with saline (solid bars). $* p=0.02$ and $p=0.006$, respectively, $t$ test.

unique research tool to study the effect of inflammationinduced preterm birth on the developing fetal brain.

IL- $1 \beta$ and TNF- $\alpha$, traditional proinflammatory cytokines, have been associated with adverse neonatal outcomes in preterm births (5). These studies sought to explore beyond these traditional proinflammatory cytokines and to elucidate the involvement of both TH1 and TH2 cytokines in the pathogenesis of inflammation-induced brain injury. The rapid activation of both a TH1 and TH2 response in fetal brains exposed to intrauterine LPS observed in this study suggest that these responses play an early and crucial role in preterm brain injury. Prolonged activation of a TH1 response is implicated in white matter damage in active inflammatory states within the CNS (31). In contrast, TH2 cytokines or agents that augment a TH2 response have been demonstrated to ameliorate disease progression $(32,33)$. Our findings of diverse upregulation of $\mathrm{TH} 1 / \mathrm{TH} 2$ pathways, at an early time point in the fetal brain, warrants further investigations into which of these mediators are essential for inducing and/or preventing neurologic injury.

The observed cytokine response in the fetal brain has been hypothesized to be secondary to a systemic fetal inflammatory response (34). In support of this hypothesis are observational studies that have demonstrated a correlation between elevated cord blood cytokine levels and the presence of histologic chorioamnionitis, as well as $\mathrm{CP}(2,35)$. Interestingly, we found that several of the TH1/TH2 mediators were more elevated in the brain than in the fetus corpus. These studies failed to demonstrate a significant correlation in the cytokine response between the different tissue compartments. While our results demonstrate that localized intrauterine inflammation can promote an immune response in the placenta, fetus, and fetal brain, our studies suggest that these responses may be specific to each tissue. Extrapolated to the clinical realm, our results suggest that cord blood and/or neonatal serum cytokine levels may be inaccurate predictors of what is occurring in the fetal brain. We hypothesize that select mediators (i.e. IL-6, CD28), elevated in the fetus/fetal liver, may be sufficient to disrupt the blood brain barrier and result in an inflammatory response that is specific to the fetal brain.

Cell death mediators have been implicated in inflammationinduced oligodendrocyte loss (13). These studies demonstrate that exposure to intrauterine LPS results in the activation of apoptotic pathways. In other animal models, the presence of histologic evidence of cell death is variable and is usually not observed until $24-48 \mathrm{~h}$ post insult $(29,30)$. Considering the early time point $(6 \mathrm{~h})$ investigated in our study, we did not expect histologic evidence of cell death.

An abnormal proliferation of astrocytes and a loss of normal white matter have been implicated as part of the mechanism of adverse neurologic outcome in the preterm neonate $(36,37)$. Inasmuch as oligodendrocyte maturation and subsequently myelination in the E15 mouse are delayed compared with the same gestational time period in the human (28-29 wk), these current studies cannot fully assess how intrauterine inflammation may affect white matter development. However, by using PLP/DM-20 as a marker of cells destined to become oligodendrocytes (38) and nestin as a marker of an immature neural phenotype $(26,39)$, these studies do suggest that exposure to intrauterine inflammation alters glial development. In fact, by using the same fetal brain specimens, these studies demonstrated that within the same brain, there is evidence of astrogliosis and a loss of pro-oligodendrocytes. These studies support the hypothesis that early disruption of astrocytes and oligodendrocytes in the preterm brain contribute to adverse neurologic outcome in preterm neonates.

In conclusion, we demonstrated that multiple signal transduction pathways are activated in the fetal brain very rapidly after exposure to intrauterine inflammation. By using a model that reproduces both intrauterine inflammation and preterm delivery, we are able to correlate these findings to human preterm delivery. These studies identify mechanisms by which brain injury occurs in the preterm neonate exposed to intrauterine inflammation. With increased understanding of these mechanisms, identification of potential targets such as $\mathrm{TH} 2$ 
cytokines, apoptotic inhibitors, and/or neurotrophins, to limit adverse neonatal outcomes can begin.

Acknowledgments. The authors thank Dr. Jerome Strauss for his advice and guidance with this research.

\section{REFERENCES}

1. Goldenberg RL, Hauth JC, Andrews WW 2000 Intrauterine infection and preterm delivery. N Engl J Med 342:1500-1507

2. Yoon BH, Romero R, Park JS, Kim M, Oh SY, Kim CJ, Jun JK 2000 The relationship among inflammatory lesions of the umbilical cord (funisitis), umbilical cord plasma interleukin 6 concentration, amniotic fluid infection, and neonatal sepsis. Am J Obstet Gynecol 183:1124-1129

3. Hagberg H, Wennerholm UB, Savman K 2002 Sequelae of chorioamnionitis. Curr Opin Infect Dis 15:301-306

4. Goncalves LF, Chaiworapongsa T, Romero R 2002 Intrauterine infection and prematurity. Ment Retard Dev Disabil Res Rev 8:3-13

5. Dammann O, Leviton A 1997 Maternal intrauterine infection, cytokines, and brain damage in the preterm newborn. Pediatr Res 42:1-8

6. DiSalvo D 1998 The correlation between placental pathology and intraventricular hemorrhage in the preterm infant. The Developmental Epidemiology Network Investigators. Pediatr Res 43:15-19

7. Rezaie P, Dean A 2002 Periventricular leukomalacia, inflammation and white matter lesions within the developing nervous system. Neuropathology 22:106-132

8. Nelson KB, Willoughby RE 2000 Infection, inflammation and the risk of cerebral palsy. Curr Opin Neurol 13:133-139

9. De Vries LS, Van Haastert IL, Rademaker KJ, Koopman C, Groenendaal F 2004 Ultrasound abnormalities preceding cerebral palsy in high-risk preterm infants. J Pediatr 144:815-820

10. Yoon BH, Park CW, Chaiworapongsa T 2003 Intrauterine infection and the development of cerebral palsy. BJOG 110:124-127

11. Ben-Hur T, Ben-Menachem O, Furer V, Einstein O, Mizrachi-Kol R, Grigoriadis N 2003 Effects of proinflammatory cytokines on the growth, fate, and motility of multipotential neural precursor cells. Mol Cell Neurosci 24:623-631

12. Balasingam V, Tejada-Berges T, Wright E, Bouckova R, Yong VW 1994 Reactive astrogliosis in the neonatal mouse brain and its modulation by cytokines. J Neurosci 14:846-856

13. Hisahara S, Okano H, Miura M 2003 Caspase-mediated oligodendrocyte cell death in the pathogenesis of autoimmune demyelination. Neurosci Res 46:387-397

14. Appel E, Kolman O, Kazimirsky G, Blumberg PM, Brodie C 1997 Regulation of GDNF expression in cultured astrocytes by inflammatory stimuli. Neuroreport 8:3309-3312

15. Moalem G, Gdalyahu A, Shani Y, Otten U, Lazarovici P, Cohen IR, Schwartz M 2000 Production of neurotrophins by activated T cells: implications for neuroprotective autoimmunity. J Autoimmun 15:331-345

16. Elovitz M, Wang Z 2004 Medroxyprogesterone acetate, but not progesterone, protects against inflammation-induced parturition and intrauterine fetal demise. Am J Obstet Gynecol 190:693-701

17. Elovitz MA, Wang Z, Chien EK, Rychlik DF, Phillippe M 2003 A new model for inflammation-induced preterm birth: the role of platelet-activating factor and Tolllike receptor-4. Am J Pathol 163:2103-2111
18. Gomez R, Romero R, Ghezzi F, Yoon BH, Mazor M, Berry SM 1998 The fetal inflammatory response syndrome. Am J Obstet Gynecol 179:194-202

19. Yang X, Yang S, Zhang J, Xue L, Hu Z 2002 Role of caspase 3 in neuronal apoptosis after acute brain injury. Chin J Traumatol 5:250-253

20. Feng Y, Fratkin JD, LeBlanc MH 2003 Inhibiting caspase-9 after injury reduces hypoxic ischemic neuronal injury in the cortex in the newborn rat. Neurosci Lett 344:201-204

21. Takashima S, Iida K, Deguchi K 1995 Periventricular leukomalacia, glial development and myelination. Early Hum Dev 43:177-184

22. Dammann O, Hagberg H, Leviton A 2001 Is periventricular leukomalacia an axonopathy as well as an oligopathy? Pediatr Res 49:453-457

23. Grinspan J 2002 Cells and signaling in oligodendrocyte development. J Neuropathol Exp Neurol 61:297-306

24. Griffiths IR, Montague P, Dickinson P 1995 The proteolipid protein gene. Neuropathol Appl Neurobiol 21:85-96

25. Peyron F, Timsit S, Thomas JL, Kagawa T, Ikenaka K, Zalc B 1997 In situ expression of PLP/DM-20, MBP, and CNP during embryonic and postnatal development of the jimpy mutant and of transgenic mice overexpressing PLP. J Neurosci Res 50:190-201

26. Duggal N, Schmidt-Kastner R, Hakim AM 1997 Nestin expression in reactive astrocytes following focal cerebral ischemia in rats. Brain Res 768:1-9

27. See J, Zhang X, Eraydin N, Mun SB, Mamontov P, Golden JA, Grinspan JB 2004 Oligodendrocyte maturation is inhibited by bone morphogenetic protein. Mol Cell Neurosci 26:481-492

28. Hirsch E, Saotome I, Hirsh D 1995 A model of intrauterine infection and preterm delivery in mice. Am J Obstet Gynecol 172:1598-1603

29. Bell MJ, Hallenbeck JM 2002 Effects of intrauterine inflammation on developing rat brain. J Neurosci Res 70:570-579

30. Debillon T, Gras-Leguen C, Verielle V, Winer N, Caillon J, Roze JC, Gressens P 2000 Intrauterine infection induces programmed cell death in rabbit periventricular white matter. Pediatr Res 47:736-742

31. Nagelkerken L 1998 Role of Th1 and Th2 cells in autoimmune demyelinating disease. Braz J Med Biol Res 31:55-60

32. Vieira PL, Heystek HC, Wormmeester J, Wierenga EA, Kapsenberg ML 2003 Glatiramer acetate (copolymer-1, copaxone) promotes Th2 cell development and increased IL-10 production through modulation of dendritic cells. J Immunol 170:4483-4488

33. Gimsa U, Wolf SA, Haas D, Bechmann I, Nitsch R 2001 Th2 cells support intrinsic anti-inflammatory properties of the brain. J Neuroimmunol 119:73-80

34. Dammann O, Kuban KC, Leviton A 2002 Perinatal infection, fetal inflammatory response, white matter damage, and cognitive limitations in children born preterm. Ment Retard Dev Disabil Res Rev 8:46-50

35. Yoon BH, Romero R, Yang SH, Jun JK, Kim IO, Choi JH, Syn HC 1996 Interleukin-6 concentrations in umbilical cord plasma are elevated in neonates with white matter lesions associated with periventricular leukomalacia. Am J Obstet Gynecol 174:1433-1440

36. Meng SZ, Arai Y, Deguchi K, Takashima S 1997 Early detection of axonal and neuronal lesions in prenatal-onset periventricular leukomalacia. Brain Dev 19:480-484

37. Kinney HC, Back SA 1998 Human oligodendroglial development: relationship to periventricular leukomalacia. Semin Pediatr Neurol 5:180-189

38. Fanarraga ML, Dickinson PJ, Sommer I, Montague P, Kyriakides E, Griffiths IR 1996 Evidence that some oligodendrocyte progenitors in the developing optic pathway express the plp gene. Glia 18:282-292

39. Clarke SR, Shetty AK, Bradley JL, Turner DA 1994 Reactive astrocytes express the embryonic intermediate neurofilament nestin. Neuroreport 5:1885-1888 